\title{
Models of strong interaction in flat-band graphene nanoribbons: Magnetic quantum crystals
}

\author{
Hao Wang and V. W. Scarola \\ Physics Department, Virginia Tech, Blacksburg, Virginia 24061, USA
}

(Received 5 August 2011; published 29 February 2012)

\begin{abstract}
Graphene-based nanostructures exhibit flat electronic energy bands in their single-particle spectrum. We consider interacting electrons in flat bands of zigzag nanoribbons. We present a protocol for flat-band projection that yields interaction-only tight-binding models. We argue that, at low densities, flat bands can delocalize single-particle basis states to support ferromagnetic quantum crystal ground states.
\end{abstract}

DOI: 10.1103/PhysRevB.85.075438

PACS number(s): 73.22.-f, 71.10.Pm

\section{INTRODUCTION}

Graphene-based structures offer unique opportunities to engineer electronic band structure by shape alone. ${ }^{1,2}$ Infinite graphene sheets exhibit a conic spectrum, but finite-sized graphene nanostructures yield a surprisingly broad array of interesting band features. A subset of graphene nanostructures reveal flat bands. Theoretical work shows that flat bands can be found, e.g., at the edges of two-dimensional graphene, ${ }^{3}$ in onedimensional graphene nanoribbons, ${ }^{3-5}$ hydrogenated graphene nanoribbons, ${ }^{6}$ graphene dots, ${ }^{7}$ and graphene antidots. ${ }^{8}$

Electrons in flat kinetic energy bands pose challenging theoretical problems. The absence of any dispersion leaves the Coulomb interaction to govern the low-energy physics. Many common approximations fail in the extreme flat-band limit. A single flat band can not lead to intraband screening as in ordinary Fermi liquids, e.g., two-dimensional graphene sheets. ${ }^{9}$ Magnetic properties in bulk graphene, in particular, occur in a regime where large screening effects (allowed by a dispersive kinetic energy) minimize the impact of the long-range intraband Coulomb interaction between electrons (see, e.g., Refs. 10-16). Flat kinetic energy bands, by contrast, do not allow screening and therefore strongly emphasize interaction effects by default. Furthermore, conventional perturbative treatments of the interaction (in comparison to the kinetic energy) fail in flat bands due to the absence of a small parameter.

Most theoretical studies of interactions in flat bands use the Hubbard model with an onsite term. ${ }^{17-19}$ The onsite Hubbard model incorporates just the energy penalty for two electrons to occupy the same site while ignoring the long-range part of the Coulomb interaction. The onsite term leads to surprising ground states in the flat-band Hubbard model. For example, work by Nagoaka ${ }^{17}$ finds ferromagnetism in flat bands at specific fillings, near one particle per site. This is in stark contrast to antiferromagnetism favored by super exchange in dispersive bands.

Graphene edges, nanoribbons, and dots present physical systems hosting flat bands. Theoretical modeling typically relies on the onsite Hubbard model to make predictions. For example, work studying flat bands in onsite Hubbard models of zigzag nanoribbons ${ }^{20,21}$ uses mean-field theory to argue for ferromagnetic states along nanoribbon edges but antiferromagnetic coupling between edges. An ab initio calculation ${ }^{22}$ and a work using both the weak-coupling renormalization group and the density-matrix renormalization group calculation ${ }^{23}$ provide similar results.
Motivated by recent experiments on graphene nanoribbons, ${ }^{24}$ we construct interacting lattice models of electrons in flat-band nanoribbons. We focus on zigzag nanoribbons because here, in contrast to arm-chair ribbons, two flat bands arise near the Fermi level even in the absence of adsorbates. ${ }^{3}$ In the top panel of Fig. 1, we schematically show a zigzag nanoribbon where $R_{0}(\sim 2.46 \AA)$ labels the width of a unit cell along the ribbon ( $x$ direction) and $L_{y}$ labels the number of zigzag chains across the ribbon ( $y$ direction). At low densities, the absence of intraband screening in flat bands suggests that the long-range part of the Coulomb interaction is relevant. We therefore construct models that include even the long-range part of the interaction. We choose to model a very specific regime: flat bands in zigzag nanoribbons because we expect the absence of conventional screening to cause flat-band electrons to order in a way that is completely distinct from electrons in bulk graphene.

The goal of our work is to establish a set of working Hamiltonians of zigzag nanoribbons. We construct a singleparticle basis of Wannier functions. We use our basis to compute the interaction matrix elements. We then establish a projection protocol that sets up approximate flat-band models. Projection into flat bands delocalizes basis states due to quantum interference. The resulting flat-band models are highly nontrivial (incorporating two bands, long-range interactions, and spin) and can lead to many quantum ground states even in the absence of significant dispersion. We make simple estimates of the low-energy properties of our models at odd denominator fillings of a single band.

We argue that, at low densities, the long-range part of the Coulomb interaction supports ferromagnetic quantum crystals (bottom panel of Fig. 1). Crystalline order projected into the flat band incorporates quantum superpositions because basis states delocalize. At low fillings, direct spin exchange leads to an effective Heisenberg model. Our simple estimates therefore predict ferromagnetic crystalline order in certain parameter regimes. Our work sets the stage for more accurate studies of our models with a general class of Jastrow-correlated wave functions that apply to flat bands. ${ }^{25}$

Our protocol differs from conventional band-structure calculations. Flat bands, in contrast to dispersive bands, are, by default, strongly interacting. Conventional applications of density functional theory accurately model the effect of core electrons while making very local approximations for the Coulomb interaction between mobile electrons. Flat bands require accurate treatment of the long-range portion of the 


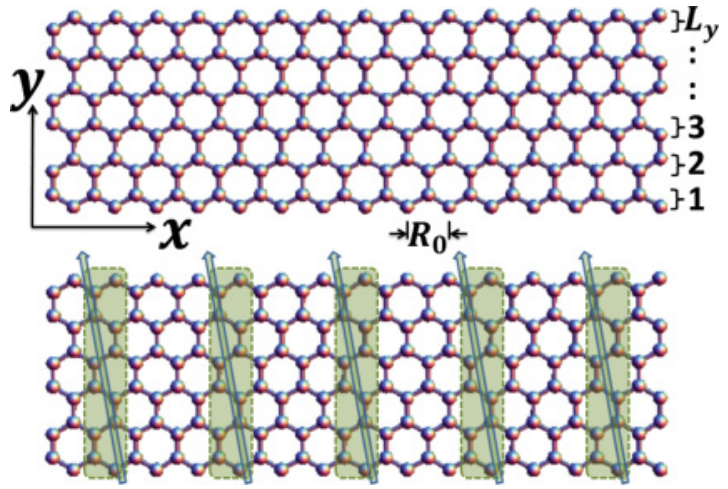

FIG. 1. (Color online) Top: Schematic of a zigzag nanoribbon of carbon atoms. Bottom: Schematic of a ferromagnetic crystal with one electron for every three unit cells in one band. The shaded areas correspond to single unit cells and the arrows indicate aligned electron spins.

unscreened Coulomb interaction between otherwise mobile electrons.

In Sec. II, we consider the band structure that arises from noninteracting tight-binding models of zigzag nanoribbons. Two flat bands are identified. In Sec. III, we construct localized single-particle basis states, orthonormal Wannier functions, from carbon $\pi_{z}$ orbitals in the honeycomb lattice model of zigzag nanoribbons. Sections IV and V use the Wannier functions to explicitly compute Coulomb interaction matrix elements for one and two flat bands, respectively. Section VI defines a projection scheme that limits the total many-body model to the flat-band portion of the single-particle spectrum. Section VII sorts terms in the many-body model to argue that, at low fillings, energetics favor ferromagnetic quantum crystals. Section VIII summarizes and looks forward to more accurate studies of the models constructed here.

\section{FLAT BANDS IN ZIGZAG GRAPHENE NANORIBBONS}

We consider interacting electrons hopping among carbon sites forming zigzag graphene nanoribbons (Fig. 1). We first model the electrons in a simple noninteracting tight-binding picture. The single-particle tight-binding Hamiltonian is ${ }^{2}$

$$
H_{0}=-t \sum_{\langle n, m\rangle}\left(\hat{c}_{n}^{\dagger} \hat{c}_{m}+\text { H.c. }\right),
$$

where the hopping integral is $t \sim 2.7 \mathrm{eV}$ for graphene ${ }^{2}$ and the sum is along bonds of the honeycomb lattice. The secondquantized operator $\hat{c}_{n}^{\dagger}$ creates a fermion at a site $n$. Labels $n$ and $m$ indicate lattice sites, in contrast to labels for unit cells, $i, j, k, l$, used in the following.

Two bands near the Fermi level flatten for large ribbon widths. ${ }^{3}$ An example band structure for a narrow width, $L_{y}=$ 4, is shown in Fig. 2. Near the Fermi surface, the conduction band (upper band, $u$ ) and valence band (lower band, $d$ ) are nearly degenerate for wave vectors $q$ in the region $q R_{0} \in$ $[2 \pi / 3,4 \pi / 3]$ and form flat bands. For larger widths, the bands flatten considerably.

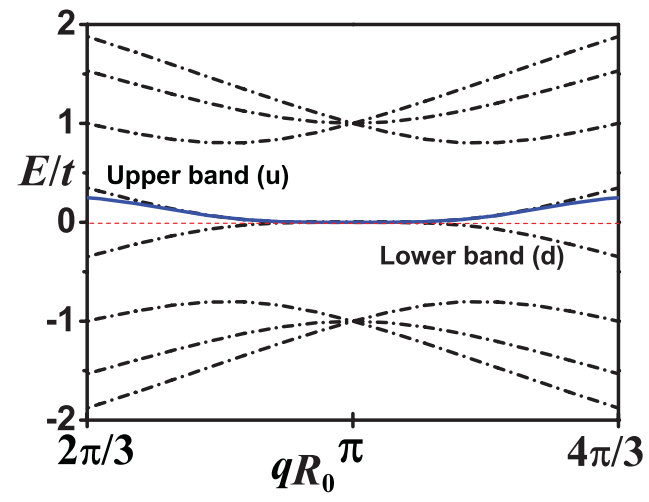

FIG. 2. (Color online) The dotted-dashed lines indicate the energy eigenvalues of Eq. (1) versus wave vector for a nanoribbon of width $L_{y}=4$ and a $q$-space mesh of $N=44$. The solid line shows the approximate expression for the energy [Eq. (3)]. Two flat bands form near $q R_{0}=\pi$. In the large- $L_{y}$ limit, the bands flatten for $2 \pi / 3 \leqslant q R_{0} \leqslant 4 \pi / 3$.

We examine the bandwidth with simple ansatz flat-band single-particle states. ${ }^{3}$ Considering states in the region $q R_{0} \in$

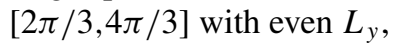

$$
\begin{aligned}
\phi_{ \pm}(q, y) & =\left[\phi_{A}(q, y), \pm \phi_{B}(q, y)\right]^{T} \\
& =\left[\left(-u_{q}\right)^{y-1}, \pm(-1)^{y-1}\left(u_{q}\right)^{L_{y}-y}\right]^{T}
\end{aligned}
$$

for $y=1, \ldots, L_{y}$ where $u_{q} \equiv 2 \cos \left(q R_{0} / 2\right)$, the energy dispersion in band $\Gamma=u, d$ can be computed analytically:

$$
\begin{aligned}
\left|E_{\Gamma}(q)\right| & \approx\left|\phi(q, y)^{T} H_{0}(q) \phi(q, y)\right| /|\phi(q, y)|^{2} \\
& =t\left(1-u_{q}^{2}\right) u_{q}^{L_{y}} /\left(1-u_{q}^{2 L_{y}}\right),
\end{aligned}
$$

with

$$
\begin{aligned}
H_{0}(q) & =t\left(\begin{array}{cc}
0 & Q(q) \\
Q^{\dagger}(q) & 0
\end{array}\right), \\
Q(q) & =\left(\begin{array}{cccc}
u_{q} & 0 & \ldots & 0 \\
1 & u_{q} & 0 & \ldots \\
\vdots & \vdots & \vdots & \vdots \\
0 & \ldots & 1 & u_{q}
\end{array}\right) .
\end{aligned}
$$

Figure 2 compares Eq. (3) with the exact results from Eq. (1).

Equation (3) can be used to determine the bandwidth. For partially filled lattices, a narrow range of single-particle basis states will be occupied. The bandwidth for states in the flatband sector vanishes for ribbons with large width:

$$
\left|E_{\Gamma}(|q-\pi| \rightarrow \pi / 3)\right| \rightarrow \frac{t}{L_{y}} .
$$

From this estimate, we see that band dispersion plays a small role for dilute ribbons with increasing ribbon widths.

A vanishing bandwidth, due to quantum interference, leaves the interaction as the dominant term in the many-body Hamiltonian for electrons. For dilute ribbons, we will work in the approximation that $H_{0}$ adds an overall constant energy shift to the spectrum. The full Hamiltonian adds the unscreened Coulomb interaction

$$
H_{\text {total }}=H_{0}+H_{V}
$$


In the following, we treat the dispersion as a small correction to the interacting term. We project the Hamiltonian into the basis of flat-band states. Our model becomes

$$
\begin{aligned}
H_{\text {total }}= & \sum_{\mathbf{q} \in \mathrm{BZ}, \sigma, \Gamma} E_{\Gamma}(q) \hat{c}_{\mathbf{q} \sigma \Gamma}^{\dagger} \hat{c}_{\mathbf{q} \sigma \Gamma}+H_{V} \\
& \rightarrow \text { constant }+\mathcal{P}_{\mathrm{FB}}^{\dagger} H_{V} \mathcal{P}_{\mathrm{FB}},
\end{aligned}
$$

where the first equality is written in terms of the creation (annihilation) operators $\hat{c}_{\mathbf{q} \sigma \Gamma}^{\dagger}\left(\hat{c}_{\mathbf{q} \sigma \Gamma}\right)$ for Bloch states at wave vector $q$ and band $\Gamma$ in the Brillouin zone (BZ), which are related to the operators for single-particle basis states by a Fourier transform

$$
\hat{c}_{j \sigma \Gamma}^{\dagger}=\frac{1}{\sqrt{N}} \sum_{\mathbf{q} \in \mathrm{BZ}} e^{i \mathbf{q} \cdot \mathbf{R}_{j}} \hat{c}_{\mathbf{q} \sigma \Gamma}^{\dagger} \cdot
$$

Here, $\mathbf{R}_{j}$ is the lattice vector of the $j$ th unit cell, $N$ defines the number of unit cells and $q$-space mesh, and $\sigma \in\{\uparrow, \downarrow\}$ denotes spin. $\mathcal{P}_{\mathrm{FB}}^{\dagger}$ denotes projection into flat bands such that the many-body eigenstates are constructed from Bloch states with $q R_{0} \in[2 \pi / 3,4 \pi / 3]$. Many-body states incorporating these values of $q$ will have essentially no kinetic energy. We consider this model as a centerpiece to understanding the electronic properties of flat-band nanoribbons at low densities.

To explore possible many-body states in zigzag nanoribbons, we construct an accurate form for Eq. (6) in the flat-band basis. We note that the absence of any dispersion excludes intraband screening as in ordinary Fermi liquids. Thus, many-body eigenstates are determined entirely by the interplay between various terms in the interaction. It is therefore crucial to accurately determine the interacting terms in Eq. (6) as prescribed by our choice of single-particle basis. To construct an accurate single-particle basis, we revisit the underlying simple tight-binding model formed from overlapping $\pi_{z}$ orbitals. We construct orthonormal Wannier functions from these orbitals. The Wannier functions will serve as single-particle basis states, allowing the construction of competing terms in a many-body model.

\section{SINGLE-PARTICLE BASIS STATES: FLAT-BAND WANNIER FUNCTIONS}

In this section, we construct a set of single-particle basis states in nanoribbon flat bands. We superpose carbon $\pi_{z}$ orbitals to form orthogonal Wannier functions. The Wannier functions will then, in later sections, be used to accurately determine interaction matrix elements.

In an isolated band, the Wannier functions are given by

$$
W_{j}(\mathbf{r})=W_{0}\left(\mathbf{r}-\mathbf{R}_{j}\right)=\frac{V}{(2 \pi)^{D}} \oint_{\mathrm{BZ}} d \mathbf{q} e^{-i \mathbf{q} \cdot \mathbf{R}_{j}} \Psi_{\mathbf{q}}(\mathbf{r}),
$$

where $D$ is the dimension, $V$ is the volume of unit cell. The Bloch functions are $\Psi_{\mathbf{q}}(\mathbf{r})=\sum_{m=1}^{M} C_{m q} \chi_{m q}(\mathbf{r})$, with $M$ atomic sites per unit cell.

To make contact with first-principles calculations on graphene nanoribbons, ${ }^{2}$ we form Bloch functions from carbon $\pi_{z}$ orbitals $\phi(\mathbf{r})=\sqrt{\xi^{5} / \pi} z e^{-\xi r}$. The basis states become $\chi_{m q}(\mathbf{r})=(1 / \sqrt{N}) \sum_{j=0}^{N-1} e^{i \mathbf{q} \cdot \mathbf{R}_{j}} \phi\left(\mathbf{r}-\mathbf{R}_{j}-\mathbf{T}_{m}\right)$, where $\mathbf{T}_{m}$ is the location of the $m$ th atom in the unit cell.
The coefficients $C_{m q}$ and energy eigenvalues $E(q)$ are obtained from diagonalization of the secular equation

$$
\left[\tilde{O}^{-1} \tilde{H}(q)\right] \mathbf{C}_{q}=E(q) \mathbf{C}_{q},
$$

where the matrix $\tilde{H}$ follows from the tight-binding Hamiltonian $H_{0}: \tilde{H}(q)_{m n}=\int d \mathbf{r} \chi_{m q}^{*}(\mathbf{r}) H_{0} \chi_{n q}(\mathbf{r})$ and the elements of the overlap matrix $\tilde{O}$ are given by $O_{m n}=\int d \mathbf{r} \chi_{m q}^{*}(\mathbf{r}) \chi_{n q}(\mathbf{r})$. The eigenvectors $\mathbf{C}_{q} \equiv\left\{C_{1 q}, \ldots, C_{M q}\right\}^{T}$ yield the coefficients used in the definition of the Wannier functions. In the tight-binding approximation, we set $O_{m n}$ proportional to the elements of the identity matrix $\delta_{m n}$.

We solve Eq. (9) to construct orthonormal Wannier functions. We consider a one-dimensional lattice of unit cells along the nanoribbon. The discrete wave vectors become $\mathbf{q}=\left(2 \pi q / N R_{0}\right) \hat{\mathbf{x}}$. The Wannier function located at $\mathbf{R}_{j}$ is then

$$
W_{j}(\mathbf{r})=\frac{1}{N} \sum_{q=0}^{N-1} e^{-i 2 \pi q j / N} \Psi_{q}(\mathbf{r}) .
$$

The Wannier functions defined in this way are unique for a $D=1$ single-band model, ${ }^{26}$ but for higher dimensions and with more bands they are not necessarily unique. ${ }^{27}$ We choose a specific set of single-particle basis states by enforcing $C_{m q}=$ $\left|C_{m q}\right|$ at the edge atomic site $m=1$. As a result, we obtain a set of real Wannier functions symmetric about the $x$ axis.

The above Wannier function can be written as a summation over all local atomic orbitals $\phi(\mathbf{r})$ located at sites $\mathbf{r}_{m i}=\mathbf{T}_{m}+$ $\mathbf{R}_{i}$. Rewriting $W$ at the origin gives

$$
W_{0}(\mathbf{r})=N_{f} \sum_{m=1}^{M} \sum_{i=0}^{N-1} \alpha_{m i} \phi\left(\mathbf{r}-\mathbf{r}_{m i}\right)
$$

with weights $\alpha_{m j}=\sum_{q=0}^{N-1} C_{m q} e^{i 2 \pi q j / N}$ and normalization constant $N_{f}$. The coefficients $\alpha$ completely determine our choice of basis.

We can extend our calculation of the Wannier functions to include both the upper and lower bands. A denser sampling in momentum space (i.e., larger $N$ ) yields more accurate Wannier functions. In practice, we find that the Wannier function has already converged when taking $N=44$ for $L_{y}=4$. The Wannier functions of upper and lower bands for the same sample ribbon are shown in Fig. 3. We note that the Wannier functions localize symmetrically about $x=0$ with an extension of less than four unit cells. The Wannier functions are also symmetric (antisymmetric) along $y$ for the upper (lower) band.

The flat-band Wannier functions constructed here correspond to a specific choice of single-particle basis. By constructing superpositions of these functions, we can equivalently construct a model using basis states localized on either edge of the ribbon via a simple rotation in the two-band space. Viewed in this way, our model implicitly includes interedge coupling in narrow ribbons because we work in the basis of $u$ and $d$ bands as opposed to a two-edge basis.

Our approach can be used to model graphene edges. Our study applies to the edge states of very wide ribbons provided we superpose our $u$ - and $d$-band Wannier functions to construct left- and right-edge Wannier functions. Our model can then be used to study edges of very wide ribbons. But, we stress that our model can not apply to the electrons in the center of 


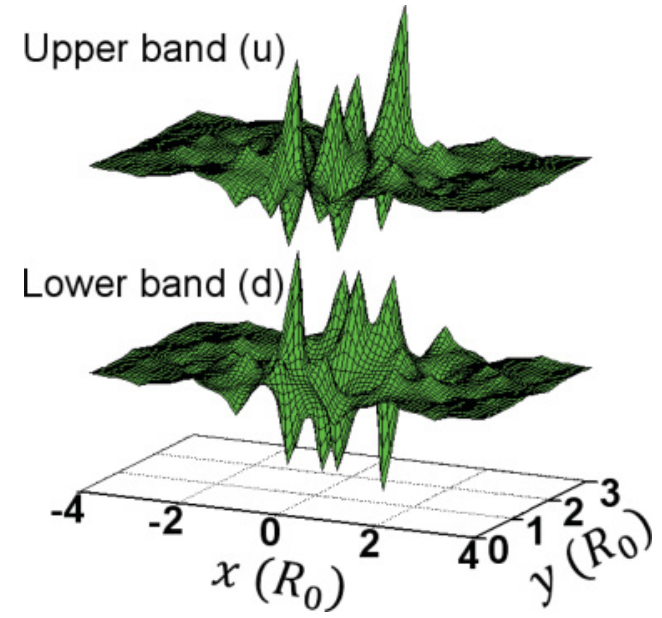

FIG. 3. (Color online) Two-dimensional Wannier functions plotted as a function of position in the lattice for a ribbon of width $L_{y}=4$. The Wannier functions tend to localize near the ribbon edges.

graphene because we have considered bands in nanoribbons that carry over only to edge states in the wide ribbon limit. (For a discussion, see Ref. 3.) In what follows, we focus on narrow ribbons and only consider Wannier functions in the $u$ and $d$-band basis.

\section{ONE-BAND COULOMB MODEL}

Interaction effects determine the low-energy properties of Eq. (5) in the absence of significant dispersion. When the chemical potential lies between the nearly flat bands of zigzag nanoribbons, the Coulomb interaction sets the dominant energy scale and mitigates response. Figure 4 shows schematic band structures for a wide ribbon with the chemical potential at the band degeneracy (left) and far from the flat-band region (right). In what follows, we focus on dilute systems corresponding to the left panel. We can, as a first approximation, assume that the valence band is inert and that only the conduction band $u$ will be active under external probes. Projection into the flat $u$ band implies that the Coulomb interaction alone operates in the massively degenerate subspace formed from $u$-band single-particle basis

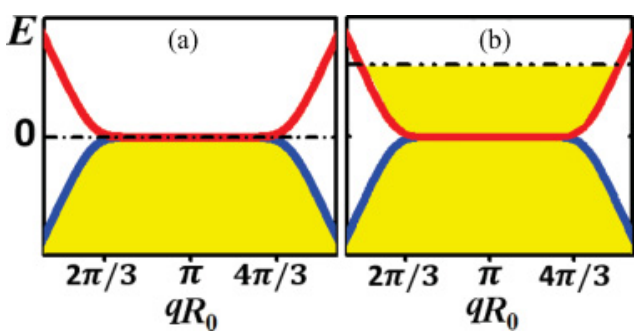

FIG. 4. (Color online) Left panel: Schematic of the energy dispersion for a wide ribbon with the Fermi level between the degenerate energy bands $u$ and $d$. In this regime, low lattice filling allows us to accurately ignore the finite dispersion near the band edges. Right panel: The same as the left panel but at larger fillings of the upper $u$ band. Here, the flat-band approximation will only be a good approximation if the Coulomb interaction is much larger than the bandwidth. states. In this section, we will consider the $u$ band only. In the following section, we will construct a model of both the $u$ and $d$ bands. band:

We consider an unscreened Coulomb interaction in a single

$$
\sum_{i, j, k, l, \sigma \sigma^{\prime}} \mathcal{V}_{i j k l} \hat{c}_{i \sigma}^{\dagger} \hat{c}_{j \sigma^{\prime}}^{\dagger} \hat{c}_{k \sigma^{\prime}} \hat{c}_{l \sigma},
$$

where the second-quantized operators $\hat{c}_{i \sigma}^{\dagger}\left(\hat{c}_{i \sigma}\right)$ create (annihilate) a fermion with spin $\sigma$ in a Wannier state centered at the $i$ th unit cell. The matrix elements $\mathcal{V}$ depend on the basis. We can rewrite the Coulomb interaction in the $u$ band in a suggestive form

$$
\begin{aligned}
H_{V}^{u}= & V_{0} \sum_{i} n_{i \uparrow} n_{i \downarrow}+\sum_{i<j} V_{i j} n_{i} n_{j}-\sum_{i<j} J_{i j} \mathbf{S}_{i} \cdot \mathbf{S}_{j} \\
& +\frac{1}{2} \sum_{\{i, j\} \nsubseteq\{k, l\}, \sigma \sigma^{\prime}} V_{i j k l} \hat{c}_{i \sigma}^{\dagger} \hat{c}_{j \sigma^{\prime}}^{\dagger} \hat{c}_{k \sigma^{\prime}} \hat{c}_{l \sigma} .
\end{aligned}
$$

Here, the single-component and total density operators are $n_{i \sigma}=\hat{c}_{i \sigma}^{\dagger} \hat{c}_{i \sigma}$ and $n_{i}=n_{i \uparrow}+n_{i \downarrow}$, respectively. The spin operators $\mathbf{S}_{i}=(1 / 2) \sum_{\sigma \sigma^{\prime}} \hat{c}_{i \sigma}^{\dagger} \tilde{\sigma}_{\sigma \sigma^{\prime}} \hat{c}_{i \sigma^{\prime}}$ are defined in terms of the Pauli matrices $\tilde{\sigma}$.

Equation (13) keeps all terms in the full Coulomb interaction. We compute the matrix elements in the basis of Wannier functions in the $u$ band. Integral equations for the coefficients are given in the Appendix [Eqs. (A1)]. The first term is the ordinary single-site Hubbard term, which is the only term that is commonly used in models of flat-band nanoribbons (see, e.g., Refs. 20 and 21). The second term captures the diagonal portion of the Coulomb interaction at long range. The absence of a dispersion implies that these terms can be relevant and must be kept in accurate models, especially at low fillings. The third term, the direct exchange term, favors ferromagnetism for $J_{i j}>0$. The last term represents remaining off-diagonal terms due to the Coulomb interaction. We find, by direct calculation, that the last terms are very small compared to the other terms for a single band.

We compute coefficients in Eq. (13) explicitly. We perform the integrals in Eqs. (A1) by approximating the exponential part of the $\pi_{z}$ orbital, $\phi(\mathbf{r})$, as a linear combination of three Gaussian functions: $\sum_{s} \gamma_{s}\left(128 \beta_{s}^{5} / \pi^{3}\right)^{1 / 4} z e^{-\beta_{s} r^{2}}$. We obtain the parameters $\gamma_{s}$ and $\beta_{s}$ from the STO-3G package. ${ }^{28}$ Data for fitting the $\pi_{z}$ orbital with $\xi=1.72$ are listed in Table I. For numerical results shown here and in the following sections, we use the Bohr radius $a_{0}=0.53 \AA$ as the unit of length and the Coulomb energy $e^{2} / 4 \pi \epsilon a_{0}(\sim 27.2 \mathrm{eV}$ in vacuum $)$ as the unit of energy.

Table II lists the coefficients computed for an $L_{y}=4$ ribbon. As we see, all coefficients are positive and can be sorted

TABLE I. Fitting parameters for the Gaussian approximation to the $\pi_{z}$ orbital with $\xi=1.72$.

\begin{tabular}{llll}
\hline \hline$s$ & \multicolumn{1}{c}{1} & \multicolumn{1}{c}{2} & \multicolumn{1}{c}{3} \\
\hline$\gamma_{s}$ & 0.15591627 & 0.60768372 & 0.39195739 \\
$\beta_{s}$ & 2.9412494 & 0.6834831 & 0.2222899 \\
\hline \hline
\end{tabular}


TABLE II. Matrix elements for one-band ( $u$ band) case for interunit-cell separations of up to $4 R_{0}$.

\begin{tabular}{lcccc}
\hline \hline \multicolumn{5}{c}{$V_{0}=2.24 \times 10^{-1}$} \\
\hline$|i-j|$ & 1 & 2 & 3 & 4 \\
$J_{i j}$ & $2.34 \times 10^{-2}$ & $4.68 \times 10^{-3}$ & $9.21 \times 10^{-4}$ & $1.69 \times 10^{-4}$ \\
$V_{i j}$ & $1.43 \times 10^{-1}$ & $9.56 \times 10^{-2}$ & $6.83 \times 10^{-2}$ & $5.23 \times 10^{-2}$ \\
\hline \hline
\end{tabular}

by $V_{0}>V_{i j}>J_{i j}>0$. The ground state can be determined by an interplay between leading terms in Eq. (13) and the chemical potential. These coefficients suggest that partially filled single bands support the formation of ferromagnetic crystals. However, the large Coulomb interaction may cause mixing between the $u$ and $d$ bands. In the next section, we construct a two-band model.

\section{TWO-BAND COULOMB MODEL}

We now consider a more comprehensive two-band model. The $u$ and $d$ bands in the flat-band region are essentially degenerate for wide ribbon widths. The Coulomb interaction can in principle favor occupancy of both bands or the occupancy of a single band. Accurate estimates of coefficients in the full two-band model will allow exploration of the two-band energy landscape to determine the band occupancy in future work.

We construct Wannier functions in both the $u$ and $d$ bands. The Hamiltonian is dominated by the following terms:

$$
\begin{aligned}
H_{V}^{u d}= & \sum_{i, \Gamma} V_{0}^{\Gamma} n_{i \Gamma \uparrow} n_{i \Gamma \downarrow}+\sum_{i}\left(V_{i i}^{\prime} n_{i u} n_{i d}-J_{i i}^{\prime} \mathbf{S}_{i u} \cdot \mathbf{S}_{i d}\right) \\
& +\sum_{i<j, \Gamma}\left(V_{i j}^{\Gamma} n_{i \Gamma} n_{j \Gamma}-J_{i j}^{\Gamma} \mathbf{S}_{i \Gamma} \cdot \mathbf{S}_{j \Gamma}\right) \\
& +\sum_{i<j} \sum_{\Gamma \neq \Gamma^{\prime}}\left(V_{i j}^{\prime} n_{i \Gamma} n_{j \Gamma^{\prime}}-J_{i j}^{\prime} \mathbf{S}_{i \Gamma} \cdot \mathbf{S}_{j \Gamma^{\prime}}\right) \\
& +\sum_{i<j} \sum_{\Gamma \neq \Gamma^{\prime}} \sum_{\sigma \sigma^{\prime}}\left(V_{i j}^{\prime \prime} \hat{c}_{i \Gamma \sigma}^{\dagger} \hat{c}_{j \Gamma^{\prime} \sigma^{\prime}}^{\dagger} \hat{c}_{j \Gamma \sigma^{\prime}} \hat{c}_{i \Gamma^{\prime} \sigma}\right. \\
& \left.+V_{i j}^{\prime \prime \prime} \hat{c}_{i \Gamma \sigma}^{\dagger} \hat{c}_{j \Gamma^{\prime} \sigma^{\prime}}^{\dagger} \hat{c}_{i \Gamma^{\prime} \sigma^{\prime}} \hat{c}_{j \Gamma \sigma}\right) .
\end{aligned}
$$

We have checked, by direct calculation, that other terms involving three and four centers are much smaller than terms kept in Eq. (14). Here, we see the Hubbard and ferromagnetic terms as in the one-band case. The last term indicates a nontrivial band-exchange term. The integrals for all coefficients are listed in the Appendix.

Equation (14) presents a central result of our work. The twoband model must be studied for different fillings and different widths to determine expected ground states. Tables III and IV show numerically computed coefficients for two example widths $L_{y}=4$ and 10 .

The tables show that the electron configurations are determined primarily by the diagonal components of the Coulomb interaction (rows 1-3). These rows are nearly equal, indicating a band symmetry, as expected. These rows govern the charge degrees of freedom. Rows 4-6 govern the spin degrees of freedom. The positive elements support ferromagnetism. The last two rows give rise to band-exchange effects.
TABLE III. Matrix elements for the two-band case with $L_{y}=4$ for inter-unit-cell separations of up to $4 R_{0}$.

\begin{tabular}{lccccc}
\hline \hline & \multicolumn{2}{c}{$V_{0}^{d}=2.28 \times 10^{-1}$} & \multicolumn{3}{c}{$V_{0}^{u}=2.24 \times 10^{-1}$} \\
& $V_{i i}^{\prime}=1.91 \times 10^{-1}$ & \multicolumn{3}{c}{$J_{i i}^{\prime}=1.32 \times 10^{-1}$} & \\
\hline$|i-j|$ & 1 & 2 & 3 & 4 & $D_{w}$ \\
$V_{i j}^{d}$ & $1.44 \times 10^{-1}$ & $9.51 \times 10^{-2}$ & $6.79 \times 10^{-2}$ & $5.21 \times 10^{-2}$ & 1.01 \\
$V_{i j}^{u}$ & $1.43 \times 10^{-1}$ & $9.56 \times 10^{-2}$ & $6.83 \times 10^{-2}$ & $5.23 \times 10^{-2}$ & 1.02 \\
$V_{i j}^{\prime}$ & $1.46 \times 10^{-1}$ & $9.56 \times 10^{-2}$ & $6.81 \times 10^{-2}$ & $5.22 \times 10^{-2}$ & 1.02 \\
$J_{i j}^{d}$ & $2.60 \times 10^{-2}$ & $3.04 \times 10^{-3}$ & $5.75 \times 10^{-4}$ & $1.09 \times 10^{-4}$ & \\
$J_{i j}^{u}$ & $2.34 \times 10^{-2}$ & $4.68 \times 10^{-3}$ & $9.21 \times 10^{-4}$ & $1.69 \times 10^{-4}$ & \\
$J_{i j}^{\prime}$ & $1.62 \times 10^{-2}$ & $3.14 \times 10^{-3}$ & $6.54 \times 10^{-4}$ & $1.27 \times 10^{-4}$ & \\
$V_{i j}^{\prime \prime}$ & $2.06 \times 10^{-2}$ & $7.44 \times 10^{-3}$ & $2.95 \times 10^{-3}$ & $1.35 \times 10^{-3}$ & \\
$V_{i j}^{\prime \prime \prime}$ & $1.05 \times 10^{-2}$ & $1.82 \times 10^{-3}$ & $3.49 \times 10^{-4}$ & $6.43 \times 10^{-5}$ & \\
\hline \hline
\end{tabular}

We construct a simple fitting form for the first three rows. We note that the coefficients $V_{i j}^{\Gamma}$ and $V_{i j}^{\prime}$ can be thought of as a softened Coulomb interaction between smeared charges located at separate unit cells $i$ and $j$. For large separations, the charges appear as point charges and interact through the Coulomb interaction, but at short ranges, our basis states smear the electron charge over the width of the ribbon. We approximate $V_{i j}^{\Gamma}$ and $V_{i j}^{\prime}$ with a convenient analytic form

$$
V_{i \neq j} \approx\left(\frac{e^{2}}{4 \pi \epsilon a_{0}}\right) \frac{a_{0} / R_{0}}{\sqrt{|i-j|^{2}+D_{w}^{2}}},
$$

where the fitting parameter $D_{w}$ is dependent on the width of the ribbon and can be determined with a numerical fitting as shown in Figs. 5 and 6. The last column of Tables III and IV shows $D_{w}$ obtained by fitting.

Equation (15) can be used to approximate the coefficients in Eq. (14) at low filling. At low filling, $V_{i j}^{\Gamma}$ and $V_{i j}^{\prime}$ determine the configuration of charges. It then suffices to consider spinexchange terms at the separations fixed by $V_{i j}^{\Gamma}$ and $V_{i j}^{\prime}$. We use this procedure to suggest possible low-energy solutions to Eq. (14).

\section{FLAT-BAND PROJECTION}

The flat-band limit, Eq. (6), establishes a unique set of nonperturbative models. In this section, we construct a set of operators that allow flat-band projection of models constructed in the previous sections. In the following section, we will then

TABLE IV. The same as Table III but for $L_{y}=10$.

\begin{tabular}{lccccc}
\hline \hline & $V_{0}^{d}=1.21 \times 10^{-1}$ & \multicolumn{3}{c}{$V_{0}^{u}=1.17 \times 10^{-1}$} \\
& $V_{i i}^{\prime}=1.03 \times 10^{-1}$ & \multicolumn{3}{c}{$J_{i i}^{\prime}=5.90 \times 10^{-2}$} & \\
\hline$|i-j|$ & 1 & 2 & 3 & 4 & $D_{w}$ \\
$V_{i j}^{d}$ & $8.93 \times 10^{-2}$ & $6.96 \times 10^{-2}$ & $5.47 \times 10^{-2}$ & $4.45 \times 10^{-2}$ & 2.09 \\
$V_{i j}^{u}$ & $8.74 \times 10^{-2}$ & $6.84 \times 10^{-2}$ & $5.41 \times 10^{-2}$ & $4.41 \times 10^{-2}$ & 2.15 \\
$V_{i j}^{\prime}$ & $9.08 \times 10^{-2}$ & $6.93 \times 10^{-2}$ & $5.44 \times 10^{-2}$ & $4.43 \times 10^{-2}$ & 2.12 \\
$J_{i j}^{d}$ & $2.82 \times 10^{-2}$ & $4.80 \times 10^{-3}$ & $1.10 \times 10^{-3}$ & $3.36 \times 10^{-4}$ & \\
$J_{i j}^{u}$ & $2.65 \times 10^{-2}$ & $6.99 \times 10^{-3}$ & $1.51 \times 10^{-3}$ & $4.30 \times 10^{-4}$ & \\
$J_{i j}^{\prime}$ & $1.65 \times 10^{-2}$ & $4.68 \times 10^{-3}$ & $1.15 \times 10^{-3}$ & $3.10 \times 10^{-4}$ & \\
$V_{i j}^{\prime \prime}$ & $1.75 \times 10^{-2}$ & $1.00 \times 10^{-2}$ & $5.82 \times 10^{-3}$ & $3.52 \times 10^{-3}$ & \\
$V_{i j}^{\prime \prime \prime}$ & $1.28 \times 10^{-2}$ & $2.85 \times 10^{-3}$ & $6.30 \times 10^{-4}$ & $1.85 \times 10^{-4}$ & \\
\hline \hline
\end{tabular}




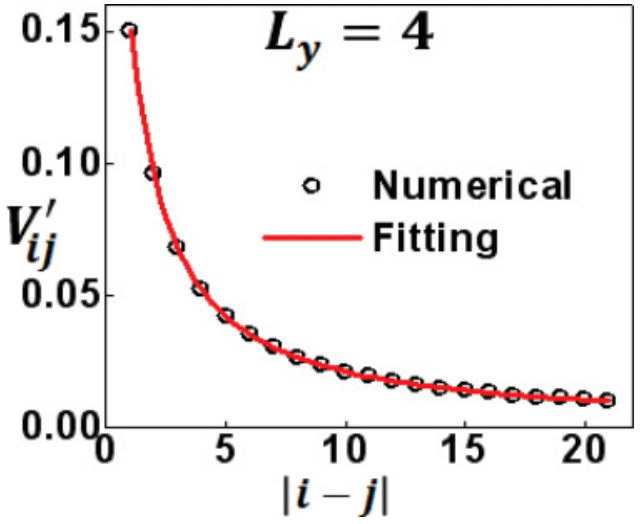

FIG. 5. (Color online) The diagonal component of the interband Coulomb interaction $\left(V_{i j}^{\prime}\right)$ for a zigzag graphene nanoribbon with $L_{y}=4$ and $N=44$. Circles are from numerical evaluation of Eqs. (A1). The solid line is a fit with Eq. (15) and $D_{w}=1.02$.

use the projected models in simple estimates of the low-energy physics.

To enforce flat-band projection, we limit all $q$-space sums to the flat-band region (FBR) $q R_{0} \in[2 \pi / 3,4 \pi / 3]$. We can therefore project into a single band by considering a flat-band operator that limits itself to the FBR:

$$
\hat{b}_{j \sigma}^{\dagger} \equiv \frac{1}{N} \sum_{l} \sum_{\mathbf{q} \in \mathrm{FBR}} e^{i \mathbf{q} \cdot\left(\mathbf{R}_{j}-\mathbf{R}_{l}\right)} \hat{c}_{l \sigma}^{\dagger} .
$$

This operator creates states centered around the unit cell at $\mathbf{R}_{j}$. We note that the states created by this operator have finite overlap with neighbors at $\mathbf{R}_{j+1}$ when the flat-band region does not encompass the entire Brillouin zone. In the limit that the flat band encompasses the entire Brillouin zone, the overlap between neighboring states vanishes and we have $\hat{b}_{j \sigma}^{\dagger} \rightarrow \hat{c}_{j \sigma}^{\dagger}$. Thus, the projection into a flat band that incorporates only a fraction of the Brillouin zone delocalizes basis states.

We can rewrite our model in terms of projected density and spin operators. The single-component and total projected density operators are $\rho_{i \sigma} \equiv \hat{b}_{i \sigma}^{\dagger} \hat{b}_{i \sigma}$ and $\rho_{i} \equiv \rho_{i \uparrow}+\rho_{i \downarrow}$, respectively. The projected spin operators are defined as

$$
\mathbf{S}_{j} \equiv \frac{1}{2 N} \sum_{\sigma \sigma^{\prime}} \sum_{\mathbf{q}, \mathbf{q}^{\prime} \in \mathrm{FBR}} e^{i\left(\mathbf{q}-\mathbf{q}^{\prime}\right) \cdot \mathbf{R}_{j}} \hat{c}_{\mathbf{q} \sigma}^{\dagger} \tilde{\boldsymbol{\sigma}}_{\sigma \sigma^{\prime}} \hat{c}_{\mathbf{q}^{\prime} \sigma^{\prime}}
$$

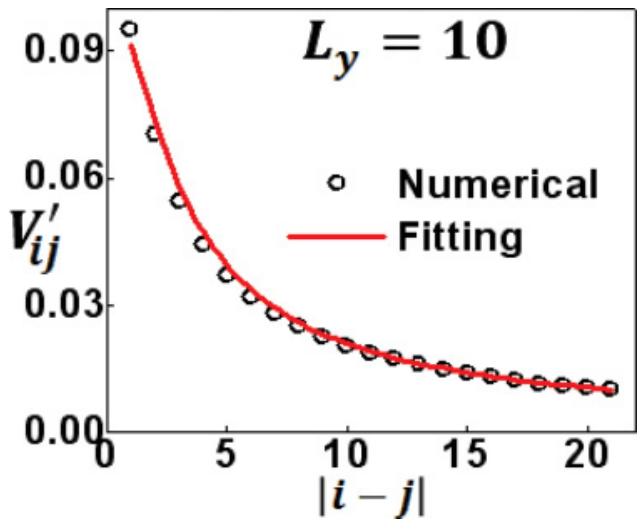

FIG. 6. (Color online) The same as Fig. 5 but for $L_{y}=10$ with $D_{w}=2.12$.
We stress that the projected operators do not exhibit ordinary commutation relations because the underlying operators create overlapping states, i.e., $\left\langle 0\left|\hat{b}_{j+1} \hat{b}_{j}^{\dagger}\right| 0\right\rangle \neq 0$.

The projected Hamiltonian can be rewritten entirely in terms of the above projected operators. Starting from an unprojected model, we impose projection using the following replacements: $c \rightarrow b, n \rightarrow \rho$, and $\mathbf{S} \rightarrow \mathbf{\$}$. For example, the flat-band projected Coulomb interaction in the $u$ band becomes

$$
\begin{aligned}
\mathcal{P}_{u}^{\dagger} H_{V}^{u} \mathcal{P}_{u}= & V_{0} \sum_{i} \rho_{i \uparrow} \rho_{i \downarrow}+\sum_{i<j} V_{i j} \rho_{i} \rho_{j}-\sum_{i<j} J_{i j} \mathbf{\$}_{i} \cdot \mathbf{S}_{j} \\
& +\frac{1}{2} \sum_{\{i, j\} \nsubseteq\{k, l\}, \sigma \sigma^{\prime}} V_{i j k l} \hat{b}_{i \sigma}^{\dagger} \hat{b}_{j \sigma^{\prime}}^{\dagger} \hat{b}_{k \sigma^{\prime}} \hat{b}_{l \sigma} \cdot
\end{aligned}
$$

The projected two-band model can also be obtained with a similar replacement applied to $H_{V}^{u d}$.

\section{LOW-ENERGY PROPERTIES}

We use flat-band projection to discuss possible low-energy states of Eq. (6) based on simple energetic arguments. A detailed quantitative analysis of low-energy states is beyond the scope of this work. We make progress by ordering terms according to dominant energy scales. We then focus on example lattice fillings.

To consider low-energy solutions of Eq. (6), we first examine the kinetic term. The kinetic term enforces a flat-band projection provided the chemical potentials lies near the flat band, i.e., Fig. 4(a). It is then sufficient to require that many-body eigenstates of $H_{V}$ utilize Bloch states with $q R_{0} \in$ $[2 \pi / 3,4 \pi / 3]$. We can analyze Eq. (14) with this $q$-space restriction by using projected operators constructed in the previous section.

We first point out an intrinsic energetic ordering to each of the terms in Eq. (14). We rewrite each of the terms according to an approximate ordering by energy and in the projected space:

$$
\begin{aligned}
\mathcal{P}_{u d}^{\dagger} H_{V}^{u d} \mathcal{P}_{u d}= & \sum_{i, \Gamma} V_{0}^{\Gamma} \rho_{i \Gamma \uparrow} \rho_{i \Gamma \downarrow} \\
& +\sum_{i, j, \Gamma, \Gamma^{\prime}}\left(\bar{V}_{i j}^{\Gamma, \Gamma^{\prime}} \rho_{i \Gamma} \rho_{j \Gamma^{\prime}}-\bar{J}_{i j}^{\Gamma, \Gamma^{\prime}} \boldsymbol{\$}_{i \Gamma} \cdot \mathbf{S}_{j \Gamma^{\prime}}\right) \\
& +H_{\text {band-exch }},
\end{aligned}
$$

where we have redefined the diagonal Coulomb terms: $\bar{V}_{i<j}^{\Gamma \neq \Gamma^{\prime}} \equiv V_{i j}^{\prime}, \bar{V}_{i i}^{\Gamma=d, \Gamma^{\prime}=u} \equiv V_{i i}^{\prime}$, and $\bar{V}_{i<j}^{\Gamma=\Gamma^{\prime}} \equiv V_{i j}^{\Gamma}$, otherwise $\bar{V}_{i j}^{\Gamma, \Gamma^{\prime}}=0$. (Note that our direct calculations find $\bar{V}_{i j}^{\Gamma \neq \Gamma^{\prime}} \approx$ $\bar{V}_{i j}^{\Gamma=\Gamma^{\prime}}$.) We have also redefined the off-diagonal exchange terms: $\bar{J}_{i<j}^{\Gamma \neq \Gamma^{\prime}} \equiv J_{i j}^{\prime}, \bar{J}_{i i}^{\Gamma=d, \Gamma^{\prime}=u} \equiv J_{i i}^{\prime}$, and $\bar{J}_{i<j}^{\Gamma=\Gamma^{\prime}} \equiv J_{i j}^{\Gamma}$, otherwise $\bar{J}_{i j}^{\Gamma, \Gamma^{\prime}}=0$. The last term in Eq. (19) corresponds to the last term in Eq. (14).

We can understand the low-energy properties of the first three terms in Eq. (19) at a few specific fillings. Considering an inert $d$ band, we assume that the $u$ band is partially filled at odd denominators, $v_{u}=1 /(2 p+1)$, where $p=1,2, \ldots$ ( $v$ indicates the number of particles per basis state). Ignoring $H_{\text {band-exch }}$ allows a decomposition of basis states into the $u$ and $d$ bands. An inert $d$ band implies that the interband interaction 
leads to an overall shift of the chemical potential. A strong external gate bias canceling this shift should be able to maintain the $u$-band filling $v_{u}=1 /(2 p+1)$.

In the limit of commuting projected density operators, it is well known ${ }^{29}$ that the first terms in Eq. (19) lead to a charge order, i.e., one-dimensional Wigner crystals with lattice spacing $2 p+1$. We therefore expect that the $u$-band electrons form a classical Wigner crystal in the limit that the flat band encompasses the entire Brillouin zone. The bottom panel of Fig. 1 depicts a classical crystal configuration in a single spin state.

In the limit that the projected density operators do not commute, the case for zigzag nanoribbons, we predict quantum crystals in partially filled bands. Quantum crystals arise, in direct analogy to Wigner crystals, as eigenstates of the projected density operators. For example, a trial quantum crystal state at $v_{u}=1 /(2 p+1)$ in spin state $\sigma$ is given by

$$
\prod_{j=0} \hat{b}_{2 p j+j, \sigma u}^{\dagger}|0\rangle \text {. }
$$

This trial state appears to minimize the energy of the first two terms in Eq. (19) by separating flat-band charges by an average of $2 p$ unit cells. Thus, the first two terms in Eq. (19) impose a rigid charge order in the $u$ band. However, the charges are significantly delocalized. A finite overlap among neighbors implies that the charges exist in a superposition of several different unit cells at once: a quantum crystal.

Provided a rigid charge ordering, we consider the next lowest-energy scale: low-energy spin properties of Eq. (19). We approximate the spin-spin coupling with an effective Heisenberg model for the $u$-band particles at $v_{u}=1 /(2 p+1)$ :

$$
H_{\mathrm{eff}}^{p}=-J_{0,2 p+1}^{u} \sum_{i} \boldsymbol{\$}_{i, u} \cdot \boldsymbol{\$}_{i+2 p+1, u} .
$$

Equation (21) applies to the case of a single band at odd denominator filling.

The ground states of Eq. (21) are ferromagnetic quantum crystals. The low-energy spin excitations are ferromagnetic magnons. The underlying rigid charge order enforces a large magnon wavelength. At $v_{u}=1 /(2 p+1)$, spin-wave theory yields excitation energies

$$
\hbar \omega_{q}=2 J_{0,2 p+1}^{u}\left\{1-\cos \left[(2 p+1) R_{0} q\right]\right\} .
$$

This dispersion offers a clear indicator of ferromagnetic crystals in the spin degrees of freedom.

At finite temperatures, the Mermin-Wagner theorem asserts that spin-spin correlations decay with a finite length scale in the one-dimensional Heisenberg model. ${ }^{30}$ Thus, ferromagnetic ordering holds only up to small length scales. The spinspin correlation length at nonzero temperatures $T$ for the Heisenberg chain with exchange coupling $J$ is ${ }^{31}$

$$
\frac{\xi_{T}}{(2 p+1) R_{0}}=\frac{A J}{4 T}\left[1+B(8 T / J)^{1 / 2} / \pi+\mathcal{O}\left(\frac{T}{J}\right)\right],
$$

where $A \approx 1.1$ and $B \approx 0.65$. Our results suggest that for $L_{y}=10$ at $T=1 \mathrm{~K}$ with $J_{i, i+3}^{u} \approx 1.5 \times 10^{-3}\left(e^{2} / 4 \pi \epsilon_{0} a_{0}\right) \approx$ $473 \mathrm{~K}$, the correlation length is $\xi_{T} /(2 p+1) R_{0} \approx 133$. Thus, about 390 unit cells containing $130 u$-band electrons are included in the formation of a fully magnetized domain at $v_{u}=1 / 3$ for these parameters.

\section{SUMMARY AND OUTLOOK}

We constructed interacting flat-band lattice models of zigzag nanoribbons. A single-particle basis of orthonormal Wannier functions was built from carbon $\pi_{z}$ orbitals in a honeycomb-ribbon lattice. The single-particle basis was used to explicitly compute the Coulomb matrix elements for two ribbon widths $L_{y}=4$ and 10. The total model [Eqs. (5) and (14)] was then projected into the flat bands of the singleparticle spectrum. The projected flat-band model [Eq. (19)] suggests ferromagnetic quantum crystal ground states.

Our flat-band model [Eq. (19)] sets the stage for more accurate analyses with a combination of numerics and many-body wave functions. The absence of a small parameter calls for a combination of variational studies and diagonalization to verify proposed ground and excited states. ${ }^{25}$ In addition to crystals discussed here, uniform quantum liquids are also possible. ${ }^{25}$

The models constructed here focus on key physics of interacting flat bands but exclude several realistic effects. In experiments on graphene nanostructures, many corrections may be required before making a detailed comparison with experiment. For example, edge roughness, defects, and substrate disorder can destroy the flat-band approximation. Furthermore, interband screening has also been ignored in the current study. While intraband screening was implicitly incorporated in our model, screening from nearby bands could lead to corrections to the pure Coulomb model studied here, e.g., RKKY-type interactions. ${ }^{32,33}$

\section{ACKNOWLEDGMENT}

We thank the T. F. Jeffress and K. M. Jeffress Memorial Trust, Grant No. J-992, for support.

\section{APPENDIX}

The coefficients in Eqs. (13) and (14) are given by

$$
\begin{aligned}
V_{0}^{\Gamma} & =\int \frac{d^{2} \mathbf{r} d^{2} \mathbf{r}^{\prime}}{\left|\mathbf{r}-\mathbf{r}^{\prime}\right|}\left|W_{0 \Gamma}(\mathbf{r}) W_{0 \Gamma}\left(\mathbf{r}^{\prime}\right)\right|^{2}, \\
J_{i j}^{\Gamma} & =2 \int \frac{d^{2} \mathbf{r} d^{2} \mathbf{r}^{\prime}}{\left|\mathbf{r}-\mathbf{r}^{\prime}\right|} W_{i \Gamma}^{*}(\mathbf{r}) W_{j \Gamma}(\mathbf{r}) W_{i \Gamma}\left(\mathbf{r}^{\prime}\right) W_{j \Gamma}^{*}\left(\mathbf{r}^{\prime}\right), \\
V_{i j}^{\Gamma} & =\int \frac{d^{2} \mathbf{r} d^{2} \mathbf{r}^{\prime}}{\left|\mathbf{r}-\mathbf{r}^{\prime}\right|}\left|W_{i \Gamma}(\mathbf{r}) W_{j \Gamma}\left(\mathbf{r}^{\prime}\right)\right|^{2}-\frac{1}{4} J_{i j}^{\Gamma}, \\
J_{i j}^{\prime} & =2 \int \frac{d^{2} \mathbf{r} d^{2} \mathbf{r}^{\prime}}{\left|\mathbf{r}-\mathbf{r}^{\prime}\right|} W_{i u}^{*}(\mathbf{r}) W_{j d}(\mathbf{r}) W_{i u}\left(\mathbf{r}^{\prime}\right) W_{j d}^{*}\left(\mathbf{r}^{\prime}\right), \\
V_{i j}^{\prime} & =\int \frac{d^{2} \mathbf{r} d^{2} \mathbf{r}^{\prime}}{\left|\mathbf{r}-\mathbf{r}^{\prime}\right|}\left|W_{i u}(\mathbf{r}) W_{j d}\left(\mathbf{r}^{\prime}\right)\right|^{2}-\frac{1}{4} J_{i j}^{\prime}, \\
V_{i j}^{\prime \prime} & =\int \frac{d^{2} \mathbf{r} d^{2} \mathbf{r}^{\prime}}{\left|\mathbf{r}-\mathbf{r}^{\prime}\right|} W_{i u}^{*}(\mathbf{r}) W_{i d}(\mathbf{r}) W_{j u}\left(\mathbf{r}^{\prime}\right) W_{j d}^{*}\left(\mathbf{r}^{\prime}\right), \\
V_{i j}^{\prime \prime \prime} & =\int \frac{d^{2} \mathbf{r} d^{2} \mathbf{r}^{\prime}}{\left|\mathbf{r}-\mathbf{r}^{\prime}\right|} W_{i u}^{*}(\mathbf{r}) W_{j u}(\mathbf{r}) W_{i d}\left(\mathbf{r}^{\prime}\right) W_{j d}^{*}\left(\mathbf{r}^{\prime}\right), \\
V_{i j k l} & =\int \frac{d^{2} \mathbf{r} d^{2} \mathbf{r}^{\prime}}{\left|\mathbf{r}-\mathbf{r}^{\prime}\right|} W_{i u}^{*}(\mathbf{r}) W_{l u}(\mathbf{r}) W_{j u}^{*}\left(\mathbf{r}^{\prime}\right) W_{k u}\left(\mathbf{r}^{\prime}\right) .
\end{aligned}
$$

The last term is used only in Eq. (13). 
${ }^{1}$ K. S. Novoselov, A. K. Geim, S. V. Morozov, D. Jiang, Y. Zhang, S. V. Dubonos, I. V. Grigorieva, and A. A. Firsov, Science 306, 666 (2004).

${ }^{2}$ A. H. Castro Neto, F. Guinea, N. M. R. Peres, K. S. Novoselov, and A. K. Geim, Rev. Mod. Phys. 81, 109 (2009).

${ }^{3}$ K. Nakada, M. Fujita, G. Dresselhaus, and M. S. Dresselhaus, Phys. Rev. B 54, 17954 (1996).

${ }^{4}$ H. H. Lin, T. Hikihara, H. T. Jeng, B. L. Huang, C. Y. Mou, and X. Hu, Phys. Rev. B 79, 035405 (2009).

${ }^{5}$ P. Potasz, A. D. Güçlü, and P. Hawrylak, Phys. Rev. B 82, 075425 (2010).

${ }^{6}$ K. Kusakabe and M. Maruyama, Phys. Rev. B 67, 092406 (2003).

${ }^{7}$ M. Ezawa, Phys. Rev. B 76, 245415 (2007); J. Fernández-Rossier and J. J. Palacios, Phys. Rev. Lett. 99, 177204 (2007); A. D. Güçlü, P. Potasz, O. Voznyy, M. Korkusinski, and P. Hawrylak, ibid. 103, 246805 (2009).

${ }^{8}$ M. Vanević, V. M. Stojanović, and M. Kindermann, Phys. Rev. B 80, 045410 (2009); J. A. Fürst, T. G. Pedersen, M. Brandbyge, and A. P. Jauho, ibid. 80, 115117 (2009).

${ }^{9}$ S. Das Sarma, E. H. Hwang, and Wang-Kong Tse, Phys. Rev. B 75, 121406(R) (2007); S. Das Sarma, S. Adam, E. H. Hwang, and E. Rossi, Rev. Mod. Phys. 83, 407 (2011).

${ }^{10}$ P. Esquinazi, D. Spemann, R. Höhne, A. Setzer, K. H. Han, and T. Butz, Phys. Rev. Lett. 91, 227201 (2003).

${ }^{11}$ N. M. R. Peres, F. Guinea, and A. H. Castro Neto, Phys. Rev. B 72, 174406 (2005).

${ }^{12}$ I. F. Herbut, Phys. Rev. Lett. 97, 146401 (2006).

${ }^{13}$ H. Ohldag, T. Tyliszczak, R. Höhne, D. Spemann, P. Esquinazi, M. Ungureanu, and T. Butz, Phys. Rev. Lett. 98, 187204 (2007).

${ }^{14}$ D. E. Sheehy and J. Schmalian, Phys. Rev. Lett. 99, 226803 (2007).
${ }^{15}$ B. L. Huang and C. Y. Mou, Europhys. Lett. 88, 68005 (2009); B. L. Huang, M. C. Chang, and C. Y. Mou, Phys. Rev. B 82, 155462 (2010)

${ }^{16}$ J. Červenka, M. I. Katsnelson, and C. F. J. Flipse, Nat. Phys. 5, 840 (2009).

${ }^{17}$ Y. Nagoaka, Phys. Rev. 147, 392 (1966).

${ }^{18}$ H. Tasaki, Prog. Theor. Phys. 99, 489 (1998).

${ }^{19}$ A. Mielke, Phys. Rev. Lett. 82, 4312 (1999).

${ }^{20}$ O. V. Yazyev, R. B. Capaz, and S. G. Louie, Phys. Rev. B 84, 115406 (2011)

${ }^{21}$ M. Fujita, K. Wakabayashi, K. Nakada, and K. Kusakabe, J. Phys. Soc. Jpn. 65, 1920 (1996).

${ }^{22}$ H. Lee, Y. W. Son, N. Park, S. Han, and J. Yu, Phys. Rev. B 72, 174431 (2005).

${ }^{23}$ T. Hikihara, X. Hu, H. H. Lin, and C. Y. Mou, Phys. Rev. B 68, 035432 (2003).

${ }^{24}$ C. Tao, L. Jiao, O. V. Yazyev, Y. C. Chen, J. Feng, X. Zhang, R. B. Capaz, J. M. Tour, A. Zettl, S. G. Louie, H. Dai, and M. F. Crommie, Nat. Phys.7, 616 (2011).

${ }^{25}$ Hao Wang and V. W. Scarola, Phys. Rev. B 83, 245109 (2011).

${ }^{26}$ W. Kohn, Phys. Rev. 115, 809 (1959).

${ }^{27}$ N. Marzari and D. Vanderbilt, Phys. Rev. B 56, 12847 (1997).

${ }^{28}$ EMSL Basis Set Exchange Library v1.2.2 at [http://bse.pnl.gov/bse/].

${ }^{29}$ J. Hubbard, Phys. Rev. B 17, 494 (1978).

${ }^{30}$ N. D. Mermin and H. Wagner, Phys. Rev. Lett. 17, 1133 (1966).

${ }^{31}$ P. Kopietz, Phys. Rev. B 40, 5194 (1989).

${ }^{32}$ L. Brey, H. A. Fertig, and S. Das Sarma, Phys. Rev. Lett. 99, 116802 (2007).

${ }^{33}$ S. Saremi, Phys. Rev. B 76, 184430 (2007). 